\title{
Qualitative analysis of wave propagation in a 3-D magnetic flux tube
}

\author{
C. Jaimes and V. Fedun \\ $\mathrm{SP}^{2} \mathrm{RC}$, Department of Applied Mathematics, University of Sheffield, Sheffield S3 7RH, UK \\ email: c.jaimes; v.fedun@sheffield.ac.uk
}

\begin{abstract}
.
In this work wave propagation in a 3-D magnetic flux tube is solved numerically. The aim is to find interaction between kink waves and higher order modes (flute modes).

A 60x60x60 cube is set up, containing a vertically oriented uniform magnetic flux tube, to solve numerically. Waves are observed propagating after triggering them with solution to the linearized system.

The waves propagate acquiring a distinctive shape (seen in the crosscut of the tube at an arbitrary height showing the radial velocity maps). It is discarded that this is caused by the existence of higher order modes and is found that the radial dependence of the phase speed creates the motion.

It was also found that the study of the profile of the radial velocity map of a slice of the system is a very intuitive way of analysing the modes of waves propagating through the flux tube.
\end{abstract}

Keywords. waves, Sun: oscillations, Sun: fluxtube

\section{Introduction}

This research is focused towards the study of the viability of wave damping through the interaction of oscillating MHD modes. Until recently many theoretical mechanisms have been put forward to explain coronal loop damping; however none of them by itself is able to account for the measured fast evanescence (within around 4 periods of oscillations). Favourite damping mechanisms are e.g. the resonant absorption, phase mixing, nonlinear interaction, wave leakage, etc. For details see e.g. Erdélyi $(2004,2007)$ and Erdélyi \& Ballai (2007).

The mechanism considered here is not really a wave damping process but the transfer of the energy from the (visible) kink wave into other higher harmonic modes that are not (yet) observed with the current high-resolution technology. The mechanism was first considered by Ruderman in an analytical study [Ruderman '92].

In the present work a $3-\mathrm{D}$ magnetic cylinder embedded in a fully ionized plasma is considered, and the full set of the non-linear, ideal MHD equations are solved numerically.

The simulation box is divided into a mesh of $60 \times 60 \times 60$, and by varying the source, that acts near the bottom end of the cylinder, the flux tube is driven(fig. 1). The source is characterized by a $B_{z}$ profile harmonically varying with time, defined by the linear solution of the system. The parameters are set satisfying the initial condition $v_{A}>c_{e}>$ $c_{k}>c_{0}>v_{A e}$ characteristic of the photosphere. Here $v_{A}$ and $v_{A e}$ are the Alfvén speeds inside and outside the cylinder, $c_{0}$ and $c_{e}$ are the sound speeds inside and outside the cylinder, and $c_{k}$ is the kink speed, respectively. 


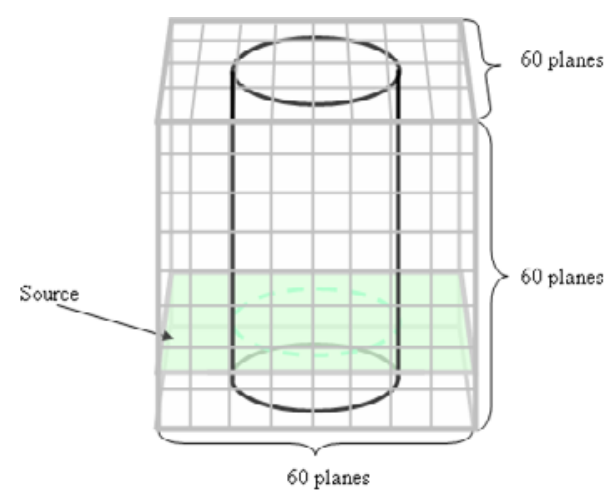

Figure 1. Graphical representation of the mesh of the computational domain showing the driver (source) obtained from the analytic solution of the linearized system.

\section{Model and Governing Equations}

The full nonlinear equations of the problem are: the continuity equation,

$$
\frac{D \rho}{D t}+\rho \nabla \cdot \mathbf{v}=0
$$

the momentum equation,

$$
\rho \frac{D \mathbf{v}}{D t}=-\nabla p+(\nabla \times \mathbf{B}) \times \frac{\mathbf{B}}{\mu},
$$

the energy equation (adiabatic case),

$$
\frac{D}{D t}\left(\frac{p}{\rho^{\gamma}}\right)=0,
$$

the ideal induction equation (with no diffusive term, i.e. in the perfectly conducting limit),

$$
\frac{\partial \mathbf{B}}{\partial t}=\nabla \times(\mathbf{v} \times \mathbf{B}),
$$

and the solenoidal equation that defines the absence of magnetic monopoles,

$$
\nabla \cdot \mathbf{B}=0
$$

It is also assumed that the plasma is fully ionized and that there is no background equilibrium flow. The linearized versions of the above set of the governing MHD equations are:

$$
\begin{gathered}
\frac{\partial \rho}{\partial t}+(\mathbf{v} \cdot \nabla) \rho_{0}+\rho_{0} \nabla \cdot \mathbf{v}=0, \\
\rho_{0} \frac{\partial \mathbf{v}}{\partial t}=-\nabla p+(\nabla \times \mathbf{B}) \times \frac{\mathbf{B}_{0}}{\mu}, \\
\frac{\partial p}{\partial t}+(\mathbf{v} \cdot \nabla) p_{0}-c_{s}^{2}\left(\frac{\partial \rho}{\partial t}+(\mathbf{v} \cdot \nabla) \rho_{0}\right)=0,
\end{gathered}
$$




$$
\begin{gathered}
\frac{\partial \mathbf{B}}{\partial t}=\nabla \times\left(\mathbf{v} \times \mathbf{B}_{0}\right), \\
\nabla \cdot \mathbf{B}=0,
\end{gathered}
$$

where $\rho, \mathbf{v}, p, \mathbf{B}$, are the small perturbations of the density $\rho_{0}$, velocity $\mathbf{v}_{0}$, pressure $p_{0}$, and magnetic field $\mathbf{B}_{0}$ respectively. Let us search for a solution of the next form (as suggested by $E \& R[E \& R$ ' 83$])$ :

$$
\Delta=\nabla \cdot \mathbf{v}=R(r) e^{i(\omega t+n \theta+k z)} .
$$

It can be shown that $R(r)$ is

for $r<a$ and,

$$
R(r)=A_{0} \begin{cases}I_{n}\left(m_{0} r\right) & \text { if } m_{0}^{2}>0 \\ J_{n} & \text { if } n_{0}^{2}=-m_{0}^{2}>0\end{cases}
$$

$$
R(r)=A_{1} K_{n}\left(m_{e} r\right) \text { provided } m_{e}^{2}>0 .
$$

for $r>a$. Here $a$ is the cylinder radius, $m_{0}$ is a quantity that charaterizes the inside of the cylinder and $m_{e}$ the outside of the cylinder and are defined as follow:

$$
m_{0}^{2}=\frac{\left(k^{2} c_{0}^{2}-\omega^{2}\right)\left(k^{2} v_{A}^{2}-\omega^{2}\right)}{\left(c_{0}^{2}+v_{A}^{2}\right)\left(k^{2} c_{T}^{2}-\omega^{2}\right)}
$$

where,

$$
c_{T}^{2}=\frac{c_{0}^{2} v_{A}^{2}}{c_{0}^{2}+v_{A}^{2}}, \quad c_{0}^{2}=\frac{\gamma p_{0}}{\rho_{0}}, \quad v_{A}^{2}=\frac{B_{0}^{2}}{\mu \rho_{0}} .
$$

The same equations apply for $m_{e}$ but with the cylinder surrounding's quantities.

The dispersion relation of surface waves of the system is:

$$
\rho_{0}\left(k^{2} v_{A}^{2}-\omega^{2}\right) m_{e} \frac{K_{n}^{\prime}\left(m_{e} a\right)}{K_{n}\left(m_{e} a\right)}=\rho_{e}\left(k^{2} v_{A e}^{2}-\omega^{2}\right) m_{0} \frac{I_{n}^{\prime}\left(m_{0} a\right)}{I_{n}\left(m_{0} a\right)},
$$

This equation is used in finding the value of the angular frequency $\omega$ for a given wave number $k$. These two parameters define the source term in the simulation.

\section{Results and Discussion}

Fig. 2 shows snapshots of radial velocity maps of horizontal crosscuts of the mesh near its top end $\left(n_{z}=50\right)$ at increasing times. Note that the kink oscillations can be inferred from the graph, and that the null velocity points form a distinctive $\mathrm{S}$ shaped line.

Fig. 3 demonstrates the kink mode $(n=1)$ with the first flute mode $(n=2)$ changing their relative phases from one image to the other. The amplitudes of the modes are chosen to be relatively similar.

The snapshots of Fig. 4 are crosscuts along $z$ (plane $n_{y}=30$ units of grid planes) and the $v_{y}$ velocity map. It can be observed that the phase speed of the waves inside the tube is greater then outside, where the waves are evanescent.

The following are some conclusions resulting from the analysis:

1. The shape of the line formed by the points of null radial velocity in snapshots of the Fig. 2 shows a clear $\mathrm{S}$ shape near the center of each snapshot. However this $\mathrm{S}$ shape cannot be seen in the snapshots of the analytical solution of the superposition of the 

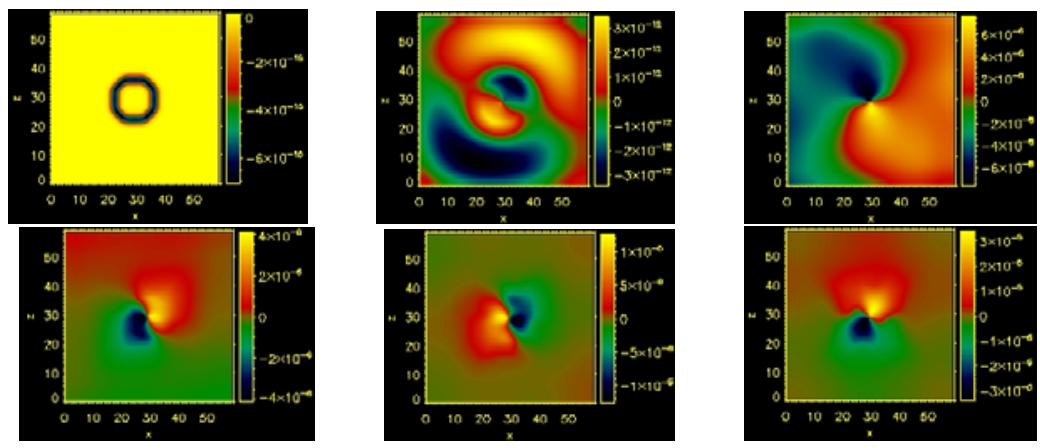

Figure 2. Radial velocity maps of the simulation at a horizontal crosscut at succesive times.
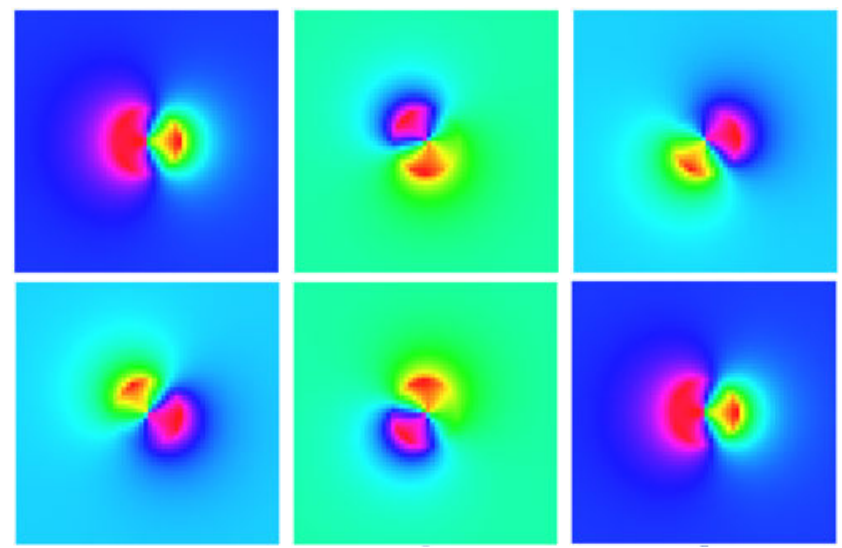

Figure 3. The radial velocity map of the kink mode also showing the first flute mode $(n=2)$ at different phases. The snapshots were calculated with the analytical solution.
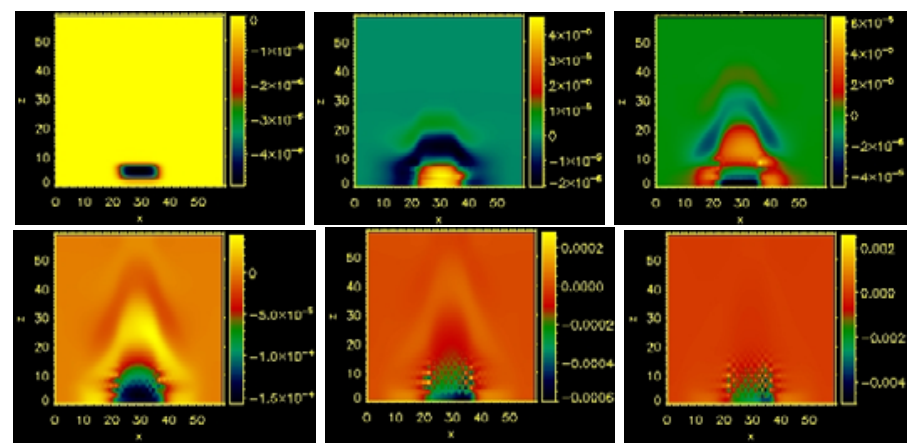

Figure 4. Vertical slices of the system showing $v_{y}$ at successive times.

modes $n=1$ and $n=2$ in Fig. 3. It can be concluded that the $\mathrm{S}$ shape may not be caused by the superposition of the kink wave with the first flute mode.

2. In Fig. 3 the radial velocity outside the tube vanishes closer to the cylinder's boundary than the radial velocity does in Fig. 2. However, it is worth to note the $v_{r}$ vanishes as slowly as it does in the numerical MHD simulation, in the map of radial velocity for analytic kink wave without adding the flute mode.

3. The $\mathrm{S}$ shape formed by the null radial velocity points in Fig. 2 can be explained 
by the radial dependence of the phase speed of the wave and the fact that the source produces a helical wave.

4. Radial velocity maps were implemented permitting to see in an easy and intuitive way the difference between a kink mode and the other higher order modes of the system.

5 . Snapshots of the analytical solution included two modes $(n=1$ and $n=2)$. The plots were constructed in such a way that the relative phases between the two modes were changed to see wether null velocity points formed and $\mathrm{S}$ shape or not. No $\mathrm{S}$ shape was found.

\section{Acknowledgements}

The authors are grateful to R. Erdélyi who has helped with his vast experience and acknowledge the financial support received from the IAU.

\section{References}

M. Aschwanden, (2004) Physics of the Solar Corona. An Introduction, Praxis Publishing Ltd. Chichester, UK

D. Banerjee, R. Erdélyi, R. Oliver \& E. O'Shea, (2007) Solar Phys., 246, 3

De Pontieu, R. Erdélyi \& S. James, (2004) Nature, 430, 536

P.M. Edwin and B. Roberts, (1983) Solar Physics, 88, 179.

R. Erdélyi, (2004), Astron. Geophys. , 45, 4.34

R. Erdélyi, (2007) AIP-CP, 919, 122

R. Erdélyi and V. Fedun, (2006) Solar Physics, 238, 41.

M. Goossens \& M.S. Ruderman, (1995) Physica Scripta, T60, 171

B. Roberts, (1981) Solar Phys., 69, 27

M.S. Ruderman, (1992) J. Plasma Phys., 47, 175

M.S. Ruderman \& B. Roberts, (2002) Apj., 577, 475

M. Terra-Homem, R. Erdélyi \& I. Ballai, (2003) Solar Phys., 217, 199

R. Erdélyi \& I. Ballai, (2007) Astrom. Nachtrichen, 328, 726 